\title{
ON PRE GENERALIZED $b$-CLOSED MAP IN TOPOLOGICAL SPACES
}

\author{
S. Sekar ${ }^{1 \S}$, R. Brindha ${ }^{2}$ \\ ${ }^{1}$ Department of Mathematics \\ Government Arts College (Autonomous) \\ Salem, 636 007, Tamil Nadu, INDIA \\ ${ }^{2}$ Department of Mathematics \\ King College of Technology \\ Namakkal, 637 020, Tamil Nadu, INDIA
}

\begin{abstract}
In this paper, we introduce a new class of pre generalized $b$-closed map in topological spaces (briefly $p g b$-closed map) and study some of their properties as well as inter relationship with other closed maps.
\end{abstract}

AMS Subject Classification: $54 \mathrm{C} 05,54 \mathrm{C} 08,54 \mathrm{C} 10$

Key Words: $\quad p g b$-closed set, $b$-closed map, $g b$-closed map, $r g b$-closed map and $g p *$-closed map

\section{Introduction}

Different types of Closed and open mappings were studied by various researchers. In 1996, Andrijevic introduced new type of set called b-open set. A.A.Omari and M.S.M. Noorani [1] introduced and studied $b$-closed map.

The aim of this paper is to introduce pre generalized $b$-closed map and to continue the study of its relationship with various generalized closed maps. Through out this paper $(X, \tau)$ and $(Y, \sigma)$ represent the non-empty topological spaces on which no separation axioms are assumed, unless otherwise mentioned.

Received: $\quad$ November 11, 2016

Revised: January 7, 2017

Published: February 28, 2017

(c) 2017 Academic Publications, Ltd. url: www.acadpubl.eu

${ }^{\S}$ Correspondence author 


\section{Preliminaries}

In this section, we referred some of the closed set definitions which was already defined by various authors.

Definition 2.1. [12] Let a subset $A$ of a topological space $(X, \tau)$, is called a pre-open set if $A \subseteq \operatorname{int}(\operatorname{cl}(A))$.

Definition 2.2. [7] Let a subset $A$ of a topological space $(X, \tau)$, is called a a semi-open set if $A \subseteq \operatorname{cl}(\operatorname{int}(A))$.

Definition 2.3. [15] Let a subset $A$ of a topological space $(X, \tau)$, is called a $\alpha$-open set if $A \subseteq \operatorname{int}(\operatorname{cl}(\operatorname{int}(A)))$.

Definition 2.4. [3] Let a subset $A$ of a topological space $(X, \tau)$, is called a $b$-open set if $A \subseteq \operatorname{cl}(\operatorname{int}(A)) \cup \operatorname{int}(\operatorname{cl}(A))$.

Definition 2.5. [6] Let a subset $A$ of a topological space $(X, \tau)$, is called a generalized closed set (briefly $g$-closed) if $\operatorname{cl}(A) \subseteq U$ whenever $A \subseteq U$ and $U$ is open in $X$.

Definition 2.6. [8] Let a subset $A$ of a topological space $(X, \tau)$, is called a generalized $\alpha$ closed set (briefly $g \alpha$ - closed) if $\alpha c l(A) \subseteq U$ whenever $A \subseteq U$ and $U$ is $\alpha$ open in $X$.

Definition 2.7. [2] Let a subset $A$ of a topological space $(X, \tau)$, is called a generalized $b$ - closed set (briefly $g b$ - closed) if $b c l(A) \subseteq U$ whenever $A \subseteq U$ and $U$ is open in $X$.

Definition 2.8. [9] Let a subset $A$ of a topological space $(X, \tau)$, is called a generalized $\alpha^{*}$-closed set (briefly g $\alpha{ }^{*}$-closed) if $\alpha \operatorname{cl}(A) \subseteq \operatorname{int} U$ whenever $A \subseteq U$ and $U$ is $\alpha$ open in $\mathrm{X}$.

Definition 2.9. [17] Let a subset $A$ of a topological space $(X, \tau)$, is called a pre-generalized closed set (briefly $p g$ - closed) if $p c l(A) \subseteq U$ whenever $A \subseteq U$ and $\mathrm{U}$ is pre-open in $X$.

Definition 2.10. [4] Let a subset $A$ of a topological space $(X, \tau)$, is called a semi generalized closed set (briefly $s g$ - closed) if $\operatorname{scl}(A) \subseteq U$ whenever $A \subseteq U$ and $U$ is semi open in $X$.

Definition 2.11. [18] Let a subset $A$ of a topological space $(X, \tau)$, is called a generalized $\alpha$ b- closed set (briefly $g \alpha b$ - closed) if $b c l(A) \subseteq U$ whenever $A \subseteq U$ and $U$ is $\alpha$ open in $X$. 
Definition 2.12. [10] Let a subset $A$ of a topological space $(X, \tau)$, is called a regular generalized b- closed set (briefly $r g b$ - closed) if $b c l(A) \subseteq U$ whenever $A \subseteq U$ and $U$ is regular open in $X$.

Definition 2.13. [13] Let a subset $A$ of a topological space $(X, \tau)$, is called pre generalized b- closed set (briefly $p g b$ - closed set) if $b c l(A) \subseteq U$ whenever $A \subseteq U$ and $U$ is pre open in $X$.

\section{On Pre Generalized b-Closed Map}

In this section, we introduce pre generalized $b$ - closed map ( $p g b$ - closed map) in topological spaces by using the notions of $p g b$ - closed sets and study some of their properties.

Definition 3.1. Let $X$ and $Y$ be two topological spaces. A map $f$ : $(X, \tau) \rightarrow(Y, \delta)$ is called pre generalized star $b$ - closed (briefly, $p g b$ - closed map) if the image of every closed set in $X$ is $p g b$-closed in $Y$.

Theorem 3.2. Every closed map is pgb-closed but not conversely.

Proof. Let $f:(X, \tau) \rightarrow(Y, \delta)$ is closed map and $V$ be an closed set in $X$ then $f(V)$ is closed in $Y$. Hence $p g b$ - closed in $Y$. Then $f$ is $p g b$ - closed.

The converse of above theorem need not be true as seen from the following example.

Example 3.3. Consider $X=Y=\{a, b, c\}, \tau=\{X, \phi,\{a, c\}\}$ and $\sigma=$ $\{Y, \phi,\{b\},\{b, c\}\}$. Let $f:(X, \tau) \rightarrow(Y, \sigma)$ be defined by $f(a)=b, f(b)=$ $c, f(c)=a$. The map is $p g b$ - closed but not closed as the image of and $\{b\}$ in $X$ is $\{c\}$ is not closed in $Y$.

Theorem 3.4. Every $b$ - closed map is pgb-closed set but not conversely.

Proof. Let $f:(X, \tau) \rightarrow(Y, \sigma)$ is $b$ - closed map and $V$ be an closed set in $X$ then $f(V)$ is closed in $Y$. Hence $p g b$ - closed in $Y$. Then $f$ is $p g b$ - closed.

The converse of above theorem need not be true as seen from the following example.

Example 3.5. Consider $X=Y=\{a, b, c\}, \tau=\{X, \phi,\{c\}\}$ and $\sigma=$ $\{Y, \phi,\{a\},\{b\},\{a, b\},\{b, c\}\}$. Let $f:(X, \tau) \rightarrow(Y, \sigma)$ be defined by $f(a)=$ $c, f(b)=a, f(c)=b$. The map is $p g b$ - closed but not $b$ - closed as the image of and $\{a, b\}$ in $X$ is $\{a, c\}$ is not $b$ - closed in $Y$. 


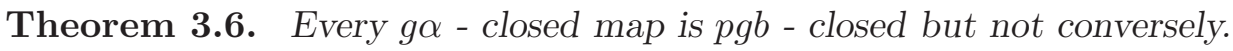

Proof. Let $f:(X, \tau) \rightarrow(Y, \sigma)$ be $g \alpha$-closed map and $V$ be an closed set in $X$ then $f(V)$ is closed in $Y$. Hence $p g b$ - closed in $Y$. Then $f$ is $p g b$-closed.

The converse of above theorem need not be true as seen from the following example.

Example 3.7. Consider $X=Y=\{a, b, c\}, \tau=\{X, \phi,\{a\},\{a, b\}\}$ and $\sigma=\{Y, \phi,\{a\},\{c\},\{a, c\}\}$. Let $f:(X, \tau) \rightarrow(Y, \sigma)$ be defined by $f(a)=$ $a, f(b)=c, f(c)=b$. The map is $p g b$ - closed but not $g \alpha$-closed as the image of $\{b, c\}$ in $X$ is $\{a, b\}$ is not $g \alpha$-closed in $Y$.

Theorem 3.8. Every $g \alpha *$ - closed map is $p g b$ - closed but not conversely.

Proof. Let $f:(X, \tau) \rightarrow(Y, \delta)$ be $g \alpha *$ - closed map and $V$ be an closed set in $X$ then $f(V)$ is closed in $Y$. Hence $p g b$ - closed in $Y$. Then $f$ is $p g b$ closed.

The converse of above theorem need not be true as seen from the following example.

Example 3.9. Consider $X=Y=\{a, b, c\}, \tau=\{X, \phi,\{b, c\}\}$ and $\sigma=$ $\{Y, \phi,\{a\},\{b\},\{a, b\}\}$. Let $f:(X, \tau) \rightarrow(Y, \sigma)$ be defined by $f(a)=b, f(b)=$ $c, f(c)=a$. The map is $p g b$ - closed but not $g \alpha *$ - closed as the image of $\{a\}$ in $X$ is $\{b\}$ is not $g \alpha *$ - closed in $Y$.

Theorem 3.10. Every $g$ - closed map is pgb-closed but not conversely.

Proof. Let $f:(X, \tau) \rightarrow(Y, \delta) g$ - closed map and $V$ be an closed set in $X$ then $f(V)$ is closed in $Y$. Hence $p g b$ - closed in $Y$. Then $f$ is $p g b$ - closed.

The converse of above theorem need not be true as seen from the following example.

Example 3.11. Consider $X=Y=\{a, b, c\}, \tau=\{X, \phi,\{a, b\}\}$ and $\sigma=$ $\{Y, \phi,\{b\},\{c\},\{b, c\}\}$. Let $f:(X, \tau) \rightarrow(Y, \sigma)$ be defined by $f(a)=c, f(b)=$ $a, f(c)=b$. The map is $p g b$-closed but not $g$-closed as the image of $\{c\}$ in $X$ is $\{b\}$ is not $g$-closed in $Y$.

Theorem 3.12. Every g $\alpha b$ - closed map is $p g b$ - closed but not conversely.

Proof. Let $f:(X, \tau) \rightarrow(Y, \sigma)$ be $g \alpha b$ - closed map and $V$ be an closed set in $X$ then $f(V)$ is closed in $Y$. Hence $p g b$ - closed in $Y$. Then $f$ is $p g b$ closed. 
The converse of above theorem need not be true as seen from the following example.

Example 3.13. Consider $X=Y=\{a, b, c\}, \tau=\{X, \phi,\{a\},\{a, c\}\}$ and $\sigma=\{Y, \phi,\{a\},\{c\},\{a, c\},\{b, c\}\}$. Let $f:(X, \tau) \rightarrow(Y, \sigma)$ be defined by $f(a)=$ $a, f(b)=c, f(c)=b$. The map is $p g b$ - closed but not $g \alpha b$ - closed as the image of and $\{b, c\}$ in $X$ is $\{b, c\}$ is not $g \alpha b$ - closed in $Y$.

Theorem 3.14. Every rgb-closed map is pgb-closed but not conversely.

Proof. Let $f:(X, \tau) \rightarrow(Y, \delta)$ be $r g b$ closed map and $V$ be an closed set in $X$ then $f(V)$ is closed in $Y$. Hence $p g b$ - closed in $Y$. Then $f$ is $p g b$ - closed.

The converse of above theorem need not be true as seen from the following example.

Example 3.15. Consider $X=Y=\{a, b, c\}, \tau=\{X, \phi,\{b, c\}\}$ and $\sigma=$ $\{Y, \phi,\{b\},\{c\},\{b, c\}\}$. Let $f:(X, \tau) \rightarrow(Y, \sigma)$ be defined by $f(a)=b, f(b)=$ $c, f(c)=a$. The map is $p g b$ - closed but not $r g b$ - closed as the image of $\{a\}$ in $X$ is $\{b\}$ is not $r g b$ - closed in $Y$.

Theorem 3.16. Every $p g b$ - closed map is pgb-closed but not conversely.

Proof. Let $f:(X, \tau) \rightarrow(Y, \delta)$ be $p g b$ - closed map and $V$ be an closed set in $X$ then $f(V)$ is closed in $Y$. Hence $g b$ - closed in $Y$. Then $f$ is $g b$ - closed.

The converse of above theorem need not be true as seen from the following example.

Example 3.17. Consider $X=Y=\{a, b, c\}, \tau=\{X, \phi,\{b\}\}$ and $\sigma=$ $\{Y, \phi,\{a\}\}$. Let $f:(X, \tau) \rightarrow(Y, \sigma)$ be defined by $f(a)=b, f(b)=c, f(c)=a$. The map is $p g$ - closed but not $p g b$ - closed as the image of $\{a, c\}$ in $X$ is $\{a, b\}$ is not $p g b$-closed in $Y$.

Theorem 3.18. Every sg-closed map is pgb-closed but not conversely.

Proof. Let $f:(X, \tau) \rightarrow(Y, \delta)$ be $s g$ - closed map and $V$ be an closed set in $X$ then $f(V)$ is closed in $Y$. Hence $p g b$ - closed in $Y$. Then $f$ is $p g b$ - closed.

The converse of above theorem need not be true as seen from the following example.

Example 3.19. Consider $X=Y=\{a, b, c\}, \tau=\{X, \phi,\{a, b\}\}$ and $\sigma=$ $\{Y, \phi,\{a, c\}\}$. Let $f:(X, \tau) \rightarrow(Y, \sigma)$ be defined by $f(a)=c, f(b)=b, f(c)=a$. The map is $p g b$-closed but not $s g$ - closed as the image of $\{c\}$ in $X$ is $\{a\}$ is not $s g$-closed in $Y$. 
Theorem 3.20. A map $f:(X, \tau) \rightarrow(Y, \sigma)$ is continuous and pgb-closed set $A$ is $p g b$-closed set of $X$ then $f(A)$ is pgb closed in $Y$.

Proof. Let $f(A) \subseteq U$ where $U$ is regular open set in $Y$. Since $f$ is continuous, $f^{-1}(U)$ is open set containing $A$. Hence $b c l(A) \subseteq f^{-1}(U)$ (as $A$ is $p g b$ - closed). Since $f$ is $p g b$ - closed $f(b c l(A)) \subseteq U$ is $p g b$ closed set $\Rightarrow \operatorname{bcl}(f(b c l(A)) \subseteq U$, Hence $b c l(A) \subseteq U$. So that $f(A)$ is $p g b$ - closed set in $Y$.

Theorem 3.21. If a map $f:(X, \tau) \rightarrow(Y, \sigma)$ is continuous and closed set and $A$ is pgb - closed then $f(A)$ is pgb - closed in $Y$.

Proof. Let $F$ be a closed set of $A$ then $F$ is $p g b$ - closed set. By theorem $3.20 f(A)$ is $p g b$ - closed. Hence $f_{A}(F)=f(F)$ is $p g b$ - closed set of $Y$. Here $f_{A}$ is $p g b$ - closed and also continuous.

Theorem 3.22. If $f:(X, \tau) \rightarrow(Y, \sigma)$ is closed map and $g:(Y, \sigma) \rightarrow(Z, \eta)$ is $p g b$ - closed map, then the composition $g \cdot f:(X, \tau) \rightarrow(Z, \eta)$ is pgb - closed map.

Proof. Let $F$ be any closed set in $(X, \tau)$. Since $f$ is closed map, $f(F)$ is closed set in $(Y, \sigma)$. Since $g$ is $p g b$ - closed map, $g(f(F))$ is $p g b$ - closed set in $(Z, \eta)$. That is $g \cdot f(F)=g(f(F))$ is $p g b$ closed. Hence $g \cdot f$ is $p g b$ closed map.

Remark 3.23. If $f:(X, \tau) \rightarrow(Y, \sigma)$ is $p g b$ - closed map and $g:(Y, \sigma) \rightarrow$ $(Z, \eta)$ is closed map, then the composition need not $p g b$ - closed map .

Theorem 3.24. A map $f:(X, \tau) \rightarrow(Y, \sigma)$ is $p g b$ - closed if and only if for each subset $S$ of $(Y, \sigma)$ and each open set $U$ containing $f^{-1}(S) \subset U$, there is a $p g b$ - open set $V$ of $(Y, \sigma)$ such that $S \subset V$ and $f^{-1}(V) \subset U$.

Proof. Suppose $f$ is $p g b$ - closed. Let $S \subset Y$ and $U$ be an open set of $(X, \tau)$ such that $f^{-1}(S) \subset U$. Now $X-U$ is closed set in $(X, \tau)$. Since $f$ is $p g b$ closed, $f(X-U)$ is $p g b$ - closed set in $(Y, \sigma)$. There fore $V=Y f(X-U)$ is an $p g b$ - open set in $(Y, \sigma)$. Now $f^{-1}(S) \subset U$ implies $S \subset V$ and $f^{-1}(V)=$ $X-f^{-1}(f(X-U)) \subset X-(X-V)=U$. (ie) $f^{-1}(V) \subset U$.

Conversely,

Let $F$ be a closed set of $(X, \tau)$. Then $f^{-1}\left(f\left(F^{c}\right)\right) \subset F^{c}$ and $F^{c}$ is an open in $(X, \tau)$. By hypothesis, there exist a $p g b$ - open set $V$ in $(Y, \sigma)$ such that $f\left(F^{c}\right) \subset V$ and $f^{-1}(V) \subset F^{c} \Rightarrow F \subset f^{-1}(V)^{c}$. Hence $V^{c} \subset f(F) \subset$ 
$f\left(\left(\left(f^{-1}(V)\right)^{c}\right)^{c} \subset V^{c} \Rightarrow f(V) \subset V^{c}\right.$. Since $V^{c}$ is $p g b$ - closed, $f(F)$ is $p g b$ closed. (ie) $f(F)$ is $p g b$ - closed in $Y$. Therefore $f$ is $p g b$ - closed.

Theorem 3.25. If $f: X_{1} \times X_{2} \rightarrow Y_{1} \times Y_{2}$ is defined as $f\left(x_{1}, x_{2}\right)=$ $\left(f_{1}\left(x_{1}\right), f_{2}\left(x_{2}\right)\right)$, then $f: X_{1} \times X_{2} \rightarrow Y_{1} \times Y_{2}$ is pgb closed map.

Proof. Let $U_{1} \times U_{2} \subset X_{1} \times X_{2}$ where $U_{i} \in \operatorname{pgbcl}\left(X_{i}\right)$, for $i=1,2$. Then $f\left(U_{1} \times U_{2}\right)=f_{1}\left(U_{1}\right) \times f_{2}\left(U_{2}\right) \in \operatorname{pgbcl}\left(X_{1} \times Y_{2}\right)$. Hence $f$ is $p g b$ - closed set.

Theorem 3.26. Let $h: X \rightarrow X_{1} \times X_{2}$ be pgb-closed map and Let $f_{i}: X \times X_{i}$ be define as $h(x)=\left(x_{1}, x_{2}\right)$ and $f_{i}(x)=x_{i}$, then $f_{i}: X \times X_{i}$ is pgb - closed map for $i=1,2$.

Proof. Let $U_{1} \times U_{2} \in X_{1} \times X_{2}$, then $f_{1}\left(U_{1}\right)=h_{1}\left(U_{1} \times X_{2}\right) \in \operatorname{pgbcl}(X)$, there fore $f_{1}$ is $p g b$ - closed. Similarly we have $f_{2}$ is $p g b$ - closed. Thus $f_{i}$ is $p g b$ - closed map for $i=1,2$.

Theorem 3.27. For any bijection map $f:(X, \tau) \rightarrow(Y, \sigma)$, the following statements are equivalent:

(i) $f^{-1}:(Y, \sigma) \rightarrow(X, \tau)$ is pgb-continuous.

(ii) $f$ is $p g b$ - open map.

(iii) $f$ is $p g b$ - closed map.

Proof. (i) $\Rightarrow$ (ii) Let $U$ be an open set of $(X, \tau)$. By assumption, $\left(f^{-1}\right)^{-1}(U)=$ $f(U)$ is $p g b$ - open in $(Y, \sigma)$ and so $f$ is $p g b$ - open.

(ii) $\Rightarrow$ (iii) Let $F$ be a closed set of $(X, \tau)$. Then $F^{c}$ is open set in $(X, \tau)$. By assumption $f\left(F^{c}\right)$ is $p g b$ - open in $(Y, \sigma)$. Therefore $f\left(F^{c}\right)=f(F)^{c}$ is $p g b$ open in $(Y, \sigma)$. That is $f(F)$ is $p g b$ - closed in $(Y, \sigma)$. Hence $f$ is $p g b$ - closed.

(iii) $\Rightarrow($ i) Let $F$ be a closed set of $(X, \tau)$. By assumption, $f(F)$ is $p g b$ - closed in $(Y, \sigma)$. But $f(F)=\left(f^{-1}\right)^{-1}(F) \Rightarrow\left(f^{-1}\right)$ is continuous. 


\section{On Pre Generalized b-Open Map}

In this section, we introduce pre generalized $b$ - open map (briefly $p g b$ - open) in topological spaces by using the notions of $p g b$ - open sets and study some of their properties.

Definition 4.1. Let $X$ and $Y$ be two topological spaces. A map $f$ : $(X, \tau) \rightarrow(Y, \delta)$ is called pre generalized $b$ - open (briefly, $p g b$ - open) if the image of every open set in $X$ is $p g b$ - open in $Y$.

Theorem 4.2. Every open map is pgb - open but not conversely.

Proof. Let $f:(X, \tau) \rightarrow(Y, \delta)$ is open map and $V$ be an open set in $X$ then $f(V)$ is open in $Y$. Hence $p g b$ - open in $Y$. Then $f$ is $p g b$ - open.

The converse of above theorem need not be true as seen from the following example.

Example 4.3. Consider $X=Y=\{a, b, c\}, \tau=\{X, \phi,\{c\}\}$ and $\sigma=$ $\{Y, \phi,\{b\},\{b, c\}\}$. Let $f:(X, \tau) \rightarrow(Y, \sigma)$ be defined by $f(a)=b, f(b)=$ $a, f(c)=c$. The map is $p g b$ - open but not open as the image of and $\{a, b\}$ in $X$ is $\{a, b\}$ is not open in $Y$.

Theorem 4.4. A map $f:(X, \tau) \rightarrow(Y, \sigma)$ is pgb-closed set if and only if for each subset $S$ of $Y$ and for each open set $U$ containing $f^{-1}(S) \subset U$ there is a pgb - open set $V$ of $Y$ such that $S \subset U$ and $f^{-1}(V) \subset U$.

Proof. Suppose $f$ is $p g b$ - closed set. Let $S \subset Y$ and $U$ be an open set of $(X, \tau)$ such that $f^{-1}(S) \subset U$. Now $X-U$ is closed set in $(X, \tau)$. Since $f$ is $p g b$ closed, $f(X-U)$ is $p g b$ closed set in $(Y, \sigma)$. Then $V=Y-f(X-U)$ is $p g b$ open set in $(Y, \sigma)$. There fore $f^{-1}(S) \subset U$ implies $S \subset V$ and $f^{-1}(V)=$ $X-f^{-1}(f(X-U)) \subset X-(X-V)=U$. (ie) $f^{-1}(V) \subset U$.

Conversely,

Let $F$ be a closed set of $(X, \tau)$. Then $f^{-1}\left(f\left(F^{c}\right)\right) \subset F^{c}$ and $F^{c}$ is an open in $(X, \tau)$. By hypothesis, there exists a pgb open set $V$ in $(Y, \sigma)$ such that $f\left(F^{c}\right) \subset V$ and $f^{-1}(V) \subset F^{c} \Rightarrow F \subset\left(f^{-1}(V)^{c}\right.$. Hence $V^{c} \subset f(F) \subset$ $f\left(\left(\left(f^{-1}(V)\right)^{c}\right)^{c}\right) \subset V^{c} \Rightarrow f(V) \subset V^{c}$. Since $V^{c} p g b$ - closed, $f(F)$ is $p g b$ closed. (ie) $f(F)$ is $p g b$ - closed in $(Y, \sigma)$ and there fore $f$ is $p g b$ - closed.

Theorem 4.5. For any bijection map $f:(X, \tau) \rightarrow(Y, \sigma)$, the following statements are equivalent.

(i) $f^{-1}:(X, \tau) \rightarrow(Y, \sigma)$ is pgb - continuous. 
(ii) $f$ is pgb open map.

(iii) $f$ is $p g b$ - closed map.

Proof. (i) $\Rightarrow$ (ii) Let $U$ be an open set of $(X, \tau)$. By assumption $\left(f^{-1}\right)^{-1}(U)=$ $f(U)$ is $p g b$ - open in $(Y, \sigma)$. There fore $f$ is $p g b$ - open map.

(ii) $\Rightarrow$ (iii) Let $F$ be closed set of $(X, \tau)$, Then $F^{c}$ is open set in $(X, \tau)$. By assumption, $f\left(F^{c}\right)$ is $p g b$ - open in $(Y, \sigma)$. There fore $f(F)$ is $p g b$ - closed in $(Y, \sigma)$. Hence $f$ is $p g b$ - closed.

(iii) $\Rightarrow$ (i) Let $F$ be a closed set of $(X, \tau)$, By assumption $f(F)$ is $p g b$ closed in $(Y, \sigma)$. But $f(F)=\left(f^{-1}\right)^{-1}(F)$. Hence $f^{-1}:(X, \tau) \rightarrow(Y, \sigma)$ is $p g b$ -continuous.

\section{References}

[1] Ahmad Al-Omari and Mohd. Salmi Md. Noorani, On Generalized b-Closed Sets. Bull. Malays. Math. Sci. Soc., (2)32(1)(2009), 19-30.

[2] D.Andrijevic, Semi-pre open sets, Mat. Vesnik., 38(1)(1986), 24-32.

[3] D.Andrijevic, On b-open sets, Mat. Vesink., 48(1-2)(1996), 59-64.

[4] Y.Gnanambal, On generalized pre - regular closed sets in topological spaces, Indian J.Pure Appl, Math 28 (1997), 351-360.

[5] D.Iyappan and N.Nagaveni, On semi generalized b - closed set, Nat. Sem. On Mat \& Comp.Sci, Jan (2010), Proc.6.

[6] N.Levine, Generalized closed sets in topology, Tend Circ., Mat. Palermo (2) 19 (1970), 89-96.

[7] N.Levine, Semi - open sets and semi - continuity in topological spaces, Amer. Math. Monthly 70 (1963)), 36-41.

[8] H.Maki, R.Devi and K.Balachandran, Associated topologies of generalized $\alpha$ - closed sets and $\alpha$ - generalized closed sets, Mem. Fac. Sci. Kochi. Univ. Ser. A.Math. 15 (1994), 51-63.

[9] H.Maki, R.J.Umehara and T.Noiri, Every topological space is pre - T 1/2, Mem. Fac. Sci. Kochi. Univ. Ser. A. Math. 17(1996), 33-42.

[10] K.Mariappa and S.Sekar, On regular generalized b-closed set, Int. Journal of Math. Analysis, 7(13), (2013), 613624 .

[11] A.S.Mashour, I.A.Hasanein and S.N.EI. Deep, $\alpha$-continuous and $\alpha$-open mapping, Acta.Math.Phys.Soc.Egypt51 (1981).

[12] A.S.Mashor Abd., M.E. El - Monsef.M.E and S.N.EI. Deep, On Pre continuous and weak pre - continuous mapping, Proc.Math.,Phys.Soc.Egypt, 53 (1982), 47-53.

[13] S.Sekar and R.Brindha, On pre generalized b- closed set in Topological Spaces, International Journal of Pure and Applied Mathematics, 111(4)(2016). 
[14] S.Sekar and K.Mariappa, On regular generalized b-Closed Map in Topological Spaces, International Journal of Mathematical Archive (IJMA), Vol.4, Issue 8, (2013), 111-116.

[15] K.Mariappa and S.Sekar, On regular generalized b-continuous map in Topological Spaces, Kyungpook Mathematical Journal, Vol.54, Issue 3, (2014), 477-483.

[16] O.Njastad, On some classes of nearly open sets, Pacific J Math., 15(1965), 961-970.

[17] J.H.Park, Y.B.Park and B.Y.Lee, On gp-closed sets and gp-continuous functions, Indian J. Pure Appl. Math., 33(1) (2002), 3-12.

[18] L. Vinayagamoorthi and N. Nagaveni, On Generalized $\alpha b$-closed set, Proceeding ICMDAllahabad, Pusbha Publication, 1 (2011). 